\begin{tabular}{cc|c}
\hline Tar. Bil. Der. & Journal of Agricultural Sciences \\
& $\begin{array}{c}\text { Dergi web sayfası: } \\
\text { www.agri.ankara.edu.tr/dergi }\end{array}$ & Journal homepage: \\
& www.agri.ankara.edu.tr/journal
\end{tabular}

\title{
Effect of Sowing Date and Humic Acid Foliar Application on Yield and Yield Components of Canola Cultivars
}

\author{
Fatemeh BAREKATI ${ }^{a}$, Eslam MAJIDI HERVAN ${ }^{a}$, Amir Hossein SHIRANI RAD ${ }^{\text {b }}$, Ghorban NOOR \\ MOHAMADI ${ }^{\mathrm{a}}$ \\ ${ }^{a}$ Department of Horticultural Science and Agronomy, Science and Research Branch, Islamic Azad University, Tehran, IRAN \\ ${ }^{\boldsymbol{b}}$ Professor of Seed and Plant Improvement Institute, AREEO, Karaj, IRAN
}

\section{ARTICLE INFO}

Research Article $\quad$ DOI: 10.15832/ankutbd.539003

Corresponding Author: Eslam MAJIDI HERVAN, E-mail: majidi_e@yahoo.com, Tel: +98 (21) 44868537

Received: 03 January 2018, Received in Revised Form: 14 March 2018, Accepted: 19 March 2018

\begin{abstract}
In order to investigate the effects of different sowing dates and humic acid foliar application on some agronomic traits of six canola cultivars a two-year experiment was carried out in 2015 and 2016 growing seasons. The experiments were laid out in a randomized complete block design arranged in factorial split plot with three replicates. The factorial combination of three sowing dates $\left(7^{\text {th }}, 17^{\text {th }}\right.$ and $27^{\text {th }}$ October $)$ and two humic acid levels $(0$ and $0.2 \%)$ were allocated to the main plots and six canola cultivars (HW118, WPN6, HL3721, L14, Tassilo and Natali) were randomized in subplots. The results indicated that the main effects of experimental factors were significant on all studied traits, except for harvest index. Interaction between sowing date and cultivar was also significant on all traits except for branch number and harvest index. In general, early seed sowing caused the highest yield and yield component as well as oil percentage and yield. Similarly, humic acid foliar application could increase agronomic traits in canola cultivars. In sum, early seed sowing and humic acid foliar application are highly recommended in canola production.
\end{abstract}

Keywords: Canola; Humic acid; Oil content; Sowing date; Yield

(C) Ankara Üniversitesi Ziraat Fakültesi

\section{Introduction}

Canola (Brassica napus L.) is one of the most important oilseed crops grown extensively in Iran. The crop has been taken into account to reduce vegetable oil import dependence. Among oilseed crops, canola has become the second largest oil crop behind soybean in the world (USDA 2013) and is widely grown for its high quality oil for human consumption. Canola seeds contain about 40 to $42 \%$ oil and its meal is also great protein supplement in cattle rations and averages approximately 35\% crude protein. Agronomic practices such as tillage, planting density, nitrogen fertilizer rate, and cultivar selection have received attention to improve survival and yield of canola (Holman et al 2011). Among these crop management practices, optimum sowing date plays key role in determining final crop yields and should be considered for the germination, seedling establishment and vegetative and reproductive stages not to be affected by unfavourable conditions (Usman et al 2016). Generally, canola should be sown before soil temperature falls under $-4{ }^{\circ} \mathrm{C}$ as 
canola is highly cold tolerant at eight leaf stage or rosette stage. In winter cropping, early sowing in autumn increases water and nutrients uptakes and vegetative growth resulting in the risk of cold tolerance loss. On the other hand, late sowing reduces total growth period and increases the risk of freezing. It has been reported that late sowing decreases biomass production, yield and yield components through increasing the risk that the crop is affected by late season drought stress (Wang et al 2012). Late sowing reduces canola vegetative growth period resulting in reduced assimilates production. In addition, late sowing increases the risk heat and drought stress during reproductive stages resulting in poor pollination, flower abortion and low seed set (Farre et al 2002). Optimum sowing date results in better seedling establishment and improved cold tolerance, which prevent cold injury and yield loss (Begna \& Angadi 2016). Reduction in oil content due to late sowing has been reported in canola by (Robertson et al 2004; Turhan et al 2011). Oil content is a trait with high heritability in canola. Oil content depends on genotype, region, soil fertility and seed age. Among environmental factors temperature is the most important factor affecting oil synthesis so that oil percentage dramatically decreases with increasing temperature (Wang et al 2012). Siliques as an active photosynthetic organ play a key role in determining seed yield. In addition, increase in silique length means more photosynthetic area and more photosynthates transfer into the seeds (Gammelvind et al 1996). Furthermore, it has been documented that seed number in siliques increases with increasing plant dry weight (Gan et al 2008). In this regard, it has been stated that canola genotypes differ to each other in terms of seed number in siliques, a trait that plays a crucial role in final seed yield (Iqbal et al 2008). However, seed number in silique is more controlled by genetic than environmental factors.

In addition to organic and mineral fertilizers, application of humic acid is getting popular in improving the crops growth and yield. Humic compounds or humus are products of decomposing plants that have complex structures and large molecular weights (Lee et al 2004). It can be extracted from any material containing welldecomposed organic matter soil, coal, composts, etc. (Sani 2014). Humic acid as the most important component of soil humus (Sparks 2003) can be applied in liquid or powder form in soil or one plant leaves (Ulukan 2008) to reduce the negative effect of environmental stresses. Humic acid has numerous benefits and all farmers across the world have come to this conclusion that humic acid is considered as an inseparable and integral part of fertilization program and soil fertility (El-Ghamry et al 2009). It has been reported that humic acid application significantly increases soil organic matter which in turn improves plant growth and development (Erik et al 2000; Hafez 2003; Abd ElAal et al 2005). In a study, humic acid application could significantly affect initial growth stages of wheat (Mirzamasoumzadeh et al 2012). Humic acid application has been advised to diminish drought stress effects, especially after flowering (Bassoa et al 2013). It has been reported that humic acid has limited promoting effect on growth, yield and quality of wheat (Delfine et al 2005). Application of $2 \%$ humic acid could increase grain and straw yield in wheat (Brunetti et al 2007).

As a result, there have been several studies on the effect of sowing date on the agronomic traits of numerous crops; however, studies on the effect of sowing date and humic acid on canola growth and production using different cultivars are still limited. Therefore, the objective of this research was to determine the effects of sowing dates and humic acid on some agronomic of six canola cultivars.

Therefore, the current study was aimed to evaluate the effects of sowing dates and humic acid foliar application on canola cultivars seed yield and yield components.

\section{Material and Methods}

In this study, the effects of different sowing date and humic acid foliar application were evaluated on plant height, branch number, seed yield and yield components, biological yield, harvest index, 
oil percentage and oil yield of six canola cultivars using a two-year experiment carried out in Seed and Plant Improvement Institute, Karaj, Iran, in 2015 and 2016 growing seasons. Meteorological data during growing season are given in Table 1. The experiments were laid out in a randomized complete block design arranged in factorial split plot with three replicates. The factorial combination of three sowing dates $\left(7^{\text {th }}, 17^{\text {th }}\right.$ and $27^{\text {th }}$ October $)$ and two humic acid levels ( 0 and $0.2 \%)$ were allocated to the main plots and six canola cultivars (HW118, WPN6, HL3721, L14, Tassilo and Natali) were randomized in sub-plots. Soil samples were collected at the depth of 0-30 and 30-60 before seed sowing, the soil physicochemical properties are presented in Table 2. According to the soil analysis results $150 \mathrm{~kg} \mathrm{ha}^{-1}$ ammonium phosphate and $150 \mathrm{~kg} \mathrm{ha}^{-1}$ potassium sulphate were applied into the soil before seed sowing. In addition, $350 \mathrm{~kg} \mathrm{ha}^{-1}$ urea was applied at three separate times $\left(100 \mathrm{~kg} \mathrm{ha}^{-1}\right.$ at sowing time, 150 $\mathrm{kg} \mathrm{ha}^{-1}$ at stem elongation and $100 \mathrm{~kg} \mathrm{ha}^{-1}$ at flowering stage). Each plot consisted of 6 ridges, $60 \mathrm{~cm}$ apart and distance between seeds on each row was $5 \mathrm{~cm}$ $\left(70,000\right.$ plant $\left.\mathrm{ha}^{-1}\right)$. Seeds were sown using a drill sower on both side of a ridge (30 cm apart) and at $25 \mathrm{~mm}$ depth. Irrigation was perfumed immediately after seed sowing. Weeds were manually controlled from 4 to 8 leaf stage. Humic acid was sprayed on plants at 4 leaf stage and stem elongation stage using backpack sprayer. Control plants were sprayed with distilled water. In order to determine plant height, branch number, silique number per plant, seed number per silique, 1000-seed weight, four central rows were manually harvested at maturity stage. Silique number in plants was determined using 10 harvested plants and average values were recorded. To determine seed number in silique, 30 siliques were randomly detached from the plants and seed number was determined. The seeds were counted and weighted using laboratory scale. Finally seed

Table 1- Meteorological data during growing season

\begin{tabular}{|c|c|c|c|c|c|c|c|c|c|c|c|}
\hline \multicolumn{12}{|c|}{ Growing season months } \\
\hline Year & Parameter & October & November & December & January & February & March & April & May & June & July \\
\hline \multirow{2}{*}{2015} & Rainfall (mm) & 13.4 & 13.7 & 31.6 & 6 & 47.8 & 21.3 & 45.4 & 2.2 & 6.6 & 0 \\
\hline & Temperature $\left({ }^{\circ} \mathrm{C}\right)$ & 18.31 & 18.2 & 6.3 & 5.2 & 7.3 & 6.7 & 13.8 & 20 & 26.4 & 30.9 \\
\hline \multirow{2}{*}{2016} & Rainfall (mm) & 3.5 & 77.4 & 28.6 & 15.6 & 8.7 & 17.8 & 75.5 & 13 & 0 & 0 \\
\hline & Temperature $\left({ }^{\circ} \mathrm{C}\right)$ & 19.4 & 10.5 & 4.6 & 5.1 & 4.9 & 11.8 & 11.7 & 19.9 & 24.2 & 28.9 \\
\hline
\end{tabular}

Table 2- Soil physicochemical properties

\begin{tabular}{lllll}
\hline & \multicolumn{2}{c}{2015} & \multicolumn{2}{c}{2016} \\
\hline Parameter & $\begin{array}{l}\text { Depth } \\
(0-30 \mathrm{~cm})\end{array}$ & $\begin{array}{l}\text { Depth } \\
(30-60 \mathrm{~cm})\end{array}$ & $\begin{array}{l}\text { Depth } \\
(0-30 \mathrm{~cm})\end{array}$ & $\begin{array}{l}\text { Depth } \\
(30-60 \mathrm{~cm})\end{array}$ \\
\hline Electrical conductivity $\left.(\mathrm{dS} \mathrm{m})^{-1}\right)$ & 1.45 & 1.24 & 1.33 & 1.15 \\
$\mathrm{pH}$ & 7.9 & 7.2 & 7.8 & 7.4 \\
Total neutralizing value (\%) & 8.56 & 6.68 & 8.25 & 8.46 \\
Moisture content (\%) & 36 & 38 & 35 & 37 \\
Organic carbon $(\%)$ & 0.91 & 0.99 & 0.83 & 0.96 \\
Total N (\%) & 0.09 & 0.07 & 0.08 & 0.06 \\
Available P $\left(\mathrm{mg} \mathrm{kh}^{-1}\right)$ & 14.7 & 15.8 & 14.2 & 15.3 \\
Available K $\left(\mathrm{mg} \mathrm{kh}^{-1}\right)$ & 197 & 155 & 165 & 148 \\
Clay $(\%)$ & 28 & 25 & 29 & 27 \\
Silt $(\%)$ & 47 & 49 & 45 & 46 \\
Sand $(\%)$ & 25 & 26 & 26 & 27 \\
Soil texture & Clay loam & Clay loam & Clay loam & Clay loam \\
\hline
\end{tabular}


yield was calculated using yield components date. Oil percentage was measured using NMR (Mq20) and oil yield was calculated through multiplying oil percentage by seed yield. In order to determine biological yield, harvested plants were sun dried, weighed and converted into kg per hectare. Harvest index was calculated as the ratio of seed yield to biological yield (Kutcher et al 2010). The data were analysed using SAS 9.0 software program. Bartlett's test showed the homogeneity of variance in all traits in both years. Therefore, combined anlaysis of variance was carried out. The Duncan's multiple range test (DMRT) was used to compare means within the combined analysis of variance.

\section{Results}

The analysis of variance indicated that the main effects of year, sowing date, humic acid foliar application and cultivar were significant on all studied traits, except for harvest index (Table 3). In addition, the results revealed that interaction between sowing date and cultivars was significant for all traits except for branch number and harvest index (Table 3). No significant interaction between sowing date and humic acid or between cultivar and humic acid were detected; therefore, only main effects are discussed. According to Table 4, sowing date significantly affected canola plant height. The highest plants were observed when seed sowing was performed on $7^{\text {th }}$ October (Table 4). The shortest plants were related to late sowing date $\left(27^{\text {th }}\right.$ October) (Table 4). Similar results were found as to branch number (Table 4). The branch number decreased when late seed sowing was practiced (Table 4). The minimum and maximum silique number was found when seed sowing was done on $27^{\text {th }}$ and $7^{\text {th }}$ October, respectively (Table 4). Seed number in silique followed a similar trend and decreased when seed sowing delayed (Table 4). 1000-seed weight decreased when seed sowing was done later than $7^{\text {th }}$ October (Table 4). In other words, the maximum 1000 -seed weight was found when seed sowing was done on $7^{\text {th }}$ October. In case of biological yield, delay in seed sowing caused a significant reduction in biological yield so that the minimum and maximum biological yield was observed when canola seeds were sown on $7^{\text {th }}$ and $27^{\text {th }}$ October, respectively (Table 4). Since seed yield is a function of interaction among yield components that are affected by sowing date, then delay in seed sowing could reduce final seed yield too (Table 4). Oil percentage and oil yield decreased as seed sowing was delayed (Table 4). As shown in Table 4, humic acid application could significantly increase plant height, branch number, yield components, biological yield, final seed yield as well as oil percentage and yield. Harvest index was the only trait that was not affected by humic acid foliar application (Table 4). The results indicated that there are significant differences among canola cultivars in terms of height, branch number yield and yield components as well as oil percentage and yield but not in terms of harvest index (Table 4). However, some cultivars were the same in terms of above mentioned traits. For example, no significant difference was found between WPN6 and Natali in terms of plant height or there was no significant difference among HW118, WPN6 and Natali cultivars in terms of final seed yield (Table 4). As mentioned before, interaction between cultivar and sowing date was found to be significant on all studied traits except for branch number and harvest index (Table 3). Comparison of means indicated that the maximum plant height was related to WPN6 and Natali cultivars when planted on $7^{\text {th }}$ October (Table 5). The highest plant height was observed in HW118, WPN6 and Natali cultivars at the $17^{\text {th }}$ October (Table 5). Similar results were found when seed sowing delayed and seeds were sown on $27^{\text {th }}$ October (Table 5). In case of silique number per plant, when WPN6 and Natali cultivars were sown on $7^{\text {th }}$ October, the maximum silique umber per plant was obtained (Table 5). On $17^{\text {th }}$ October, the maximum silique number was related to HW118 cultivar (Table 5). The maximum silique number per plant was related to HW118 and WPN6 cultivars when sown on $27^{\text {th }}$ October (Table $5)$. The maximum seed number in silique was related to WPN6 and Natali cultivars sown on $7^{\text {th }}$ October (Table 5), however, when seed sowing was performed $17^{\text {th }}$ or $27^{\text {th }}$ October, the maximum seed number was observed in HW118, WPN6 and Natali 
cultivars (Table 5). Almost in all sowing dates, the maximum 1000-seed weight was related to HW118, WPN6 and Natali cultivars (Table 5). The maximum biological yield was related to WPN6 and Natali cultivars when sown on $7^{\text {th }}$ October (Table 5).
Seed sowing on $17^{\text {th }}$ October caused the maximum biological yield in HW118, WPN6 and Natali cultivars (Table 5$)$. Late sowing date ( $27^{\text {th }}$ October) decreased biological yield in all the cultivars (Table 5). Almost in all sowing dates, the maximum seed

Table 3- Analysis of variance on yield and yield components of canola cultivars as affected by sowing date and humic acid application

\begin{tabular}{|c|c|c|c|c|c|c|c|c|c|c|c|}
\hline S.O.V & $D f$ & $\begin{array}{l}\text { Plant } \\
\text { height }\end{array}$ & $\begin{array}{l}\text { Branch } \\
\text { number }\end{array}$ & $\begin{array}{l}\text { Silique } \\
\text { number }\end{array}$ & $\begin{array}{l}\text { Seed number } \\
\text { in silique }\end{array}$ & $\begin{array}{l}\text { 1000-seed } \\
\text { weight }\end{array}$ & $\begin{array}{l}\text { Biological } \\
\text { yield }\end{array}$ & $\begin{array}{l}\text { Seed } \\
\text { yield }\end{array}$ & $\begin{array}{l}\text { Harvest } \\
\text { index }\end{array}$ & $\begin{array}{l}\text { Oil } \\
\text { percentage }\end{array}$ & $\begin{array}{l}\text { Oil } \\
\text { yield }\end{array}$ \\
\hline $\bar{Y}$ & 1 & $* *$ & $* *$ & ** & $* *$ & $* *$ & $* *$ & $* *$ & ns & $* *$ & $* *$ \\
\hline $\mathrm{E}$ & 4 & 23.66 & 0.69 & 356.49 & 1.81 & 0.38 & 2076708 & 1497597.80 & 33.97 & 0.26 & 291703.43 \\
\hline S & 2 & $* *$ & $* *$ & $* *$ & $* *$ & $* *$ & $* *$ & $* *$ & ns & $* *$ & $* *$ \\
\hline $\mathrm{Y} \times \mathrm{S}$ & 2 & ns & ns & $*$ & * & $* *$ & ns & ns & ns & ns & ns \\
\hline $\mathrm{H}$ & 1 & $* *$ & $* *$ & $* *$ & $* *$ & $* *$ & $* *$ & $* *$ & ns & $* *$ & $* *$ \\
\hline $\mathrm{Y} \times \mathrm{H}$ & 1 & ns & ns & ns & ns & ns & ns & ns & ns & $\mathrm{ns}$ & $\mathrm{ns}$ \\
\hline $\mathrm{S} \times \mathrm{H}$ & 2 & ns & ns & ns & ns & ns & ns & ns & ns & ns & ns \\
\hline $\mathrm{Y} \times \mathrm{S} \times \mathrm{H}$ & 2 & ns & ns & ns & ns & $\mathrm{ns}$ & ns & ns & ns & ns & ns \\
\hline E & 20 & 38.14 & 0.82 & 112.84 & 1.32 & 0.28 & 912493 & 255431.20 & 12.30 & 0.26 & 50708.73 \\
\hline $\mathrm{C}$ & 5 & $* *$ & $* *$ & $* *$ & $* *$ & $* *$ & $* *$ & $* *$ & ns & $* *$ & $* *$ \\
\hline $\mathrm{Y} \times \mathrm{C}$ & 5 & ns & ns & ns & ns & ns & ns & ns & ns & ns & ns \\
\hline $\mathrm{S} \times \mathrm{C}$ & 10 & $* *$ & ns & $* *$ & $* *$ & $* *$ & $* *$ & $* *$ & ns & $* *$ & $* *$ \\
\hline $\mathrm{Y} \times \mathrm{S} \times \mathrm{C}$ & 10 & ns & ns & ns & ns & ns & ns & ns & ns & ns & ns \\
\hline $\mathrm{H} \times \mathrm{C}$ & 5 & ns & ns & ns & $\mathrm{ns}$ & $\mathrm{ns}$ & $\mathrm{ns}$ & $\mathrm{ns}$ & ns & ns & ns \\
\hline $\mathrm{Y} \times \mathrm{H} \times \mathrm{C}$ & 5 & ns & ns & ns & ns & ns & ns & ns & ns & ns & ns \\
\hline $\mathrm{S} \times \mathrm{H} \times \mathrm{C}$ & 10 & ns & ns & ns & ns & ns & ns & ns & ns & ns & $\mathrm{ns}$ \\
\hline $\mathrm{Y} \times \mathrm{S} \times \mathrm{H} \times \mathrm{C}$ & 10 & ns & ns & ns & ns & ns & ns & ns & ns & ns & ns \\
\hline$\underline{E}$ & 120 & 51.80 & 1.39 & 110.09 & 1.30 & 0.17 & 1122770 & 235235.30 & 12.12 & 0.13 & 40811.81 \\
\hline C.V (\%) & & 4.94 & 18.18 & 6.77 & 6.87 & 9.51 & 6.27 & 11.98 & 14.55 & 0.89 & 11.92 \\
\hline
\end{tabular}

S.O.V, source of variation; df, degree of freedom; Y, year; E, error; P, sowing date; H, humic acid; C, cultivar; C.V, coefficient of variation; *,** and $\mathrm{ns}$ indicate significance at $\mathrm{P}<0.05,0.01$ and not significant, respectively

Table 4- Main effects of sowing date, humic acid and cultivar on yield and yield components

\begin{tabular}{|c|c|c|c|c|c|c|c|c|c|c|c|}
\hline Factors & Levels & $\begin{array}{l}\text { Plant } \\
\text { height } \\
(\mathrm{cm})\end{array}$ & $\begin{array}{l}\text { Branch } \\
\text { number }\end{array}$ & $\begin{array}{l}\text { Silique } \\
\text { number }\end{array}$ & $\begin{array}{l}\text { Seed } \\
\text { number } \\
\text { in silique }\end{array}$ & $\begin{array}{l}\text { 1000-seed } \\
\text { weight } \\
\text { (g) }\end{array}$ & $\begin{array}{l}\text { Biological } \\
\text { yield } \\
\left(\mathrm{kg} \mathrm{ha}^{-1}\right)\end{array}$ & $\begin{array}{l}\text { Seed } \\
\text { yield } \\
\left(\mathrm{kg} \mathrm{ha}^{-1}\right)\end{array}$ & $\begin{array}{l}\text { Harvest } \\
\text { index (\%) }\end{array}$ & $\begin{array}{l}\text { Oil } \\
\text { percentage }\end{array}$ & $\begin{array}{l}\text { Oil yield } \\
\left(k g h a^{-1}\right)\end{array}$ \\
\hline \multirow{3}{*}{$\begin{array}{l}\text { Sowing } \\
\text { date }\end{array}$} & October $7^{\text {th }}$ & $173.92 \mathrm{a}$ & $8.63 a$ & $215.41 \mathrm{a}$ & $19.80 \mathrm{a}$ & $5.47 \mathrm{a}$ & $21835 \mathrm{a}$ & $5243 a$ & $24.06 \mathrm{a}$ & $42.87 \mathrm{a}$ & $2250 \mathrm{a}$ \\
\hline & October $17^{\text {th }}$ & $144.43 b$ & $6.40 \mathrm{~b}$ & $151.09 \mathrm{~b}$ & $16.62 b$ & $4.41 \mathrm{~b}$ & $16689 b$ & $4069 b$ & $24.41 \mathrm{a}$ & $41.57 \mathrm{~b}$ & $1694 b$ \\
\hline & October $27^{\text {th }}$ & $118.19 \mathrm{c}$ & $4.42 \mathrm{c}$ & $98.24 \mathrm{c}$ & $13.40 \mathrm{c}$ & $3.30 \mathrm{c}$ & $12148 \mathrm{c}$ & $2826 \mathrm{c}$ & $23.29 \mathrm{a}$ & $40.25 \mathrm{c}$ & $1140 \mathrm{c}$ \\
\hline \multirow{2}{*}{$\begin{array}{l}\text { Humic } \\
\text { acid }\end{array}$} & - & $142.12 b$ & $6.21 \mathrm{~b}$ & $147.21 \mathrm{~b}$ & $16.21 \mathrm{~b}$ & $4.26 \mathrm{~b}$ & $16229 b$ & $3894 b$ & $23.93 a$ & $41.38 \mathrm{~b}$ & $1624 b$ \\
\hline & + & $148.90 \mathrm{a}$ & $6.75 \mathrm{a}$ & $162.62 \mathrm{a}$ & $17.00 \mathrm{a}$ & $4.53 \mathrm{a}$ & $17553 a$ & $4199 a$ & $23.91 \mathrm{a}$ & $41.75 \mathrm{a}$ & $1765 a$ \\
\hline \multirow{6}{*}{ Cultivars } & HW118 & $148.21 b$ & $6.76 \mathrm{a}$ & $159.75 b$ & $17.02 \mathrm{a}$ & $4.53 a$ & $17261 b$ & $4198 \mathrm{a}$ & $24.36 \mathrm{a}$ & $41.75 b$ & $1759 a$ \\
\hline & WPN6 & $152.42 \mathrm{a}$ & $7.03 \mathrm{a}$ & $170.1 \mathrm{a}$ & $17.46 \mathrm{a}$ & $4.68 \mathrm{a}$ & $18353 a$ & $4355 a$ & $23.79 \mathrm{a}$ & $41.94 \mathrm{a}$ & $1840 \mathrm{a}$ \\
\hline & HL3721 & $140.65 \mathrm{c}$ & $6.08 \mathrm{~b}$ & $144.37 \mathrm{c}$ & $16.01 \mathrm{~b}$ & $4.216 \mathrm{~b}$ & $15924 \mathrm{c}$ & $3832 b$ & $24.00 \mathrm{a}$ & $41.318 \mathrm{c}$ & $1595 b$ \\
\hline & L14 & $141.08 \mathrm{c}$ & $6.09 \mathrm{~b}$ & $145.6 \mathrm{c}$ & $16.03 b$ & $4.217 \mathrm{~b}$ & $16069 \mathrm{c}$ & $3834 b$ & $23.74 \mathrm{a}$ & $41.311 \mathrm{c}$ & $1598 b$ \\
\hline & Tassilo & $138.94 \mathrm{c}$ & $5.93 b$ & $140.72 \mathrm{c}$ & $15.78 b$ & $4.12 b$ & $15608 \mathrm{c}$ & $3736 b$ & $23.76 \mathrm{a}$ & $41.19 \mathrm{c}$ & $1554 \mathrm{~b}$ \\
\hline & Natali & $151.76 \mathrm{a}$ & $7.00 \mathrm{a}$ & $168.95 \mathrm{a}$ & $17.34 \mathrm{a}$ & $4.62 \mathrm{a}$ & $18130 \mathrm{a}$ & $4321 \mathrm{a}$ & $23.87 \mathrm{a}$ & $41.88 \mathrm{ab}$ & $1823 \mathrm{a}$ \\
\hline
\end{tabular}

In each column same letters show that there are no significantly difference according to DMRT 
Table 5- Interaction between sowing date and cultivar on some agronomic traits of canola

\begin{tabular}{|c|c|c|c|c|c|c|c|c|c|c|c|c|}
\hline & \multicolumn{2}{|l|}{$\begin{array}{l}\text { Plant height } \\
(\mathrm{cm})\end{array}$} & \multicolumn{4}{|c|}{$\begin{array}{l}\text { Silique number } \\
\text { per plant }\end{array}$} & \multicolumn{3}{|c|}{$\begin{array}{l}\text { Seed number } \\
\text { in silique }\end{array}$} & \multicolumn{3}{|c|}{$\begin{array}{l}\text { 1000-seed weight } \\
(\mathrm{g})\end{array}$} \\
\hline & $7^{\text {th }}$ & $17^{t h}$ & $27^{\text {th }}$ & $7^{t h}$ & $17^{t h}$ & $27^{t h}$ & $7^{t h}$ & $17^{t h}$ & $27^{\text {th }}$ & $7^{\text {th }}$ & $17^{\text {th }}$ & $27^{\text {th }}$ \\
\hline HW118 & $165.05 \mathrm{c}$ & $154.07 \mathrm{a}$ & $125.51 \mathrm{a}$ & $194.99 d$ & $170.31 \mathrm{a}$ & $113.95 \mathrm{a}$ & $18.89 b$ & $17.73 \mathrm{a}$ & $14.45 \mathrm{a}$ & $5.14 b$ & $4.72 \mathrm{a}$ & $3.72 \mathrm{a}$ \\
\hline WPN6 & $184.51 \mathrm{a}$ & $149.33 \mathrm{a}$ & $123.42 \mathrm{a}$ & $240.57 \mathrm{a}$ & $160.24 b$ & $109.50 \mathrm{ab}$ & $21.12 \mathrm{a}$ & $17.11 \mathrm{a}$ & $14.15 \mathrm{a}$ & $5.87 \mathrm{a}$ & $4.55 \mathrm{ab}$ & $361 \mathrm{a}$ \\
\hline HL 3721 & $167.81 b c$ & $139.68 b$ & $114.46 b$ & $231.87 \mathrm{~cd}$ & $141.35 \mathrm{c}$ & $90.84 \mathrm{c}$ & $19.11 b$ & $16.04 b$ & $12.87 \mathrm{~b}$ & $5.27 \mathrm{~b}$ & $4.25 \mathrm{bc}$ & $3.12 b$ \\
\hline L14 & $173.03 b$ & $137.45 b$ & $112.78 b$ & $206.37 b$ & $136.40 \mathrm{~cd}$ & $86.55 \mathrm{~cd}$ & $19.63 b$ & $15.83 b$ & $12.62 b$ & $5.34 b$ & $4.21 \mathrm{c}$ & $3.00 \mathrm{~b}$ \\
\hline Tassilo & $170.70 \mathrm{bc}$ & $134.57 \mathrm{~b}$ & $111.54 \mathrm{~b}$ & $200.92 b c$ & $132.90 \mathrm{~d}$ & $82.90 \mathrm{~d}$ & $19.38 b$ & $15.56 \mathrm{~b}$ & $12.40 \mathrm{~b}$ & $5.34 b$ & $4.12 \mathrm{c}$ & $2.91 \mathrm{~b}$ \\
\hline \multirow[t]{3}{*}{ Natali } & $182.40 \mathrm{a}$ & $151.47 \mathrm{a}$ & $121.40 \mathrm{a}$ & $235.76 \mathrm{a}$ & $165.35 b$ & $105.72 b$ & $20.70 \mathrm{a}$ & $17.45 \mathrm{a}$ & $13.89 \mathrm{a}$ & $5.80 \mathrm{a}$ & $4.61 \mathrm{a}$ & $3.46 \mathrm{a}$ \\
\hline & \multicolumn{2}{|c|}{$\begin{array}{l}\text { Biological yield } \\
\left(\mathrm{kg} \mathrm{ha}^{-1}\right)\end{array}$} & & \multicolumn{2}{|l|}{$\begin{array}{l}\text { Seed yield } \\
\left(\mathrm{kg} \mathrm{ha}^{-1}\right)\end{array}$} & & \multicolumn{2}{|c|}{$\begin{array}{l}\text { Oil } \\
\text { percentage }\end{array}$} & & \multicolumn{2}{|l|}{$\begin{array}{l}\text { Oil yield } \\
\left(\mathrm{kg} \mathrm{ha}^{-1}\right)\end{array}$} & \\
\hline & $7^{\text {th }}$ & $17^{\text {th }}$ & $27^{\text {th }}$ & $7^{\text {th }}$ & $17^{\text {th }}$ & $27^{\text {th }}$ & $7^{\text {th }}$ & $17^{t h}$ & $27^{\text {th }}$ & $7^{\text {th }}$ & $17^{\text {th }}$ & $27^{\text {th }}$ \\
\hline HW118 & $19943.20 \mathrm{~d}$ & $18189.30 \mathrm{a}$ & $13649.80 \mathrm{a}$ & $4892.90 \mathrm{~b}$ & $4452.90 \mathrm{a}$ & $3249.20 \mathrm{a}$ & $42.48 \mathrm{c}$ & $42.00 \mathrm{a}$ & $40.75 a$ & $2080.40 b$ & $1871.80 \mathrm{a}$ & $1324.70 \mathrm{a}$ \\
\hline WPN6 & $24321.30 \mathrm{a}$ & $17534.80 \mathrm{a}$ & $13201.60 \mathrm{ab}$ & $5672.10 \mathrm{a}$ & $4284.10 \mathrm{a}$ & $3110.10 \mathrm{a}$ & $43.41 \mathrm{a}$ & $41.77 \mathrm{a}$ & $40.63 \mathrm{ab}$ & $2464.00 \mathrm{a}$ & $1790.70 \mathrm{a}$ & $1264.70 \mathrm{a}$ \\
\hline HL3721 & $20487.50 \mathrm{~cd}$ & $15897.50 \mathrm{~b}$ & $11387.90 \mathrm{c}$ & $5003.70 \mathrm{~b}$ & $3870.50 \mathrm{~b}$ & $2622.80 b$ & $42.58 \mathrm{bc}$ & $41.35 b$ & $40.01 \mathrm{c}$ & $2132.08 b$ & $1601.70 \mathrm{~b}$ & $1051.08 \mathrm{~b}$ \\
\hline L14 & $21536.90 \mathrm{~b}$ & $15556.40 \mathrm{bc}$ & $11115.00 \mathrm{c}$ & $5186.70 b$ & $3786.70 \mathrm{~b}$ & $2527.30 \mathrm{~b}$ & $42.78 b$ & $41.26 \mathrm{~b}$ & $39.88 \mathrm{c}$ & $2219.30 b$ & $1565.08 \mathrm{~b}$ & $1008.90 \mathrm{~b}$ \\
\hline Tassilo & $20982.20 \mathrm{bc}$ & $15120.80 \mathrm{c}$ & $10720.30 \mathrm{c}$ & $5117.10 \mathrm{~b}$ & $3674.90 \mathrm{~b}$ & $2415.50 b$ & $42.68 b c$ & $41.14 b$ & $39.76 \mathrm{c}$ & $2184.90 b$ & $1513.80 \mathrm{~b}$ & $962.50 \mathrm{~b}$ \\
\hline Natali & $23740.90 \mathrm{a}$ & $17834.30 \mathrm{a}$ & $12813.50 \mathrm{~b}$ & $5584.20 \mathrm{a}$ & $4344.30 \mathrm{a}$ & $3033.30 \mathrm{a}$ & $43.28 \mathrm{a}$ & $41.90 \mathrm{a}$ & $40.46 b$ & $2417.50 \mathrm{a}$ & $1821.10 \mathrm{a}$ & $1228.80 \mathrm{a}$ \\
\hline
\end{tabular}

In each column same letters show that there are no significantly difference according to DMRT

yield was obtained from HW118, WPN6 and Natali cultivars (Table 5). When canola seeds were sown on $7^{\text {th }}$ October, WPN6 and Natali cultivars showed the maximum oil percentage (Table 5). When seed sowing was performed on $17^{\text {th }}$ October, HW118, WPN6 and Natali cultivars showed the maximum oil percentage and in late sowing date $\left(27^{\text {th }}\right.$ October $)$ HW118 and WPN6 cultivars indicated maximum oil percentage (Table 5). Almost in all sowing dates, the maximum oil yield, was obtained from HW118, WPN6 and Natali cultivars (Table 5).

\section{Discussion}

The results indicated that sowing date has a significant effect on yield and yield components, biological yield, oil percentage and oil yield in canola cultivars. These results agree with previous reports on canola yield (Farre et al 2002; Kutcher et al 2010). A similar experiment carried out in the United State (Holman et al 2011), documented the advantage of optimum sowing date in canola, and showed that optimum sowing date can vary across a relatively short geographical distance, largely driven by substantial differences in annual precipitation and elevation. In another study it has also been reported that sowing date significantly affects winter survival, suggesting early sowing can assure sufficient canola plant growth to survive the winter (Holman et al 2011; Darby et al 2013), but sowing too early also can have negative effects on plant. The reduction in canola seed yield due to delay in seed sowing has been reported by several authors (Robertson et al 2004; Faraji et al 2009). The increase in canola seed yield due to early sowing date might be attributed to more light, water and nutrients absorption by plants thus, increasing photosynthetic capacity. These results are in agreement with those of Chauhan et al (1993). Sowing date is a critical factor that controls growing season length and hence, final seed yield. Early sowing postpones flowering which is an important factor leading to the highest yields (Jenkins \& Leitch 1986). Jansinka et al (1989) indicated that seed and oil yields decreased with delay in sowing date. The differences between canola cultivars in terms of seed yield might be attributed to their differences in growth traits such as branches number that mirrored differences in yield components and hence, increased seed yield. Sharief \& Keshta (2000) have found similar results. According to the results, the effect of humic acid foliar application was significant on plant height, 
branch number, seed yield and yield components as well as biological yield, oil percentage and yield. It has been reported that humic acid application reduces the requirement of other fertilizers (Sani 2014). For instance, previous findings have shown that total chemical contents percentage in leaves of cucumber plants due to humic acid application (ElNemr et al 2012).

The increase in plant height on account of humic acid application may be due to enhanced shoot growth. The results are in line with (Salwa \& Eisa 2011) who stated that maximum plant height was recorded when $15 \mathrm{~kg} \mathrm{ha}^{-1}$ humic acid was applied. Increase in branch number due to humic acid can also be attributed to increased plant growth as reported by Sani (2014). Regarding increase in silique number on account of humic acid application, similar results have been reported with regard to the increased number of pod in soya bush (Farnia 2006). Furthermore, in a study carried out by Hagh-Parast et al (2012), humic acid application caused noticeable increase in number of pod in chickpea. Since humic acid causes remarkable increase in photosynthesis activity (Saadati \& Baghi 2014), therefore, more flowers will be formed in canola plant which is effective on formation of fertile silique and seed production. It has been documented that humic acid application leads to increased photosynthesis rate and consequently, assimilates. In the same direction, assimilates retransfer rate would increase and seed weight will be increased (Farnia \& Nasrollahi 2010). Similar results were reported by Wang et al (2015) that humic acid application increases 1000seed weight and biological yield. Rao et al (2000) also reported such results in case of increased dry matter yields of mustard due to humic acid application. In this study, humic acid application caused an increase in oil percentage and oil yield in comparison with control plants. These results are in agreement with the report of Rajpar et al (2011) which showed that application of humic acid had significant effect on oil percentage and yield. Similarly, it has been reported that foliar application of humic acid improved seed yield and oil content in mustard (Chris et al 2005). Generally, humates enhance nutrient uptake and increase the yield and quality of various oilseed crops (MacCarthy et al 2001; Salt et al 2001).

\section{Conclusions}

The canola cultivars responded to different sowing dates. Growth, seed yield and oil yield decreased with delayed sowing date. By contrast, early seed sowing could improve canola yield and oil production. In this experiment, application of humic acid could significantly increase yield and yield components of canola as well as oil percentage and oil yield. Therefore, choosing a suitable sowing date (as early as possible) is essential depending upon growing conditions and cultivar. According to this experiment, it can be suggested that the most appropriate sowing time for a desire seed yield in the experimental region is early October and humic acid foliar application as a way to increase canola yield and production is recommended.

\section{References}

Abd El-Aal F S, Shafeek M R, Ahmed A A \& Shaheen A M (2005). Response of growth and yield of onion plants to potassium fertilizer and humic acid. Journal of Agricultural Science Mansoura University 30(1): 315-326

Bassoa B, Cammarano D, Fiorentino C \& Ritchie J (2013). Wheat yield response to spatially variable nitrogen fertilizer in Mediterranean environment. European Journal of Agronomy 51: 65-70

Begna S H \& Angadi S V (2016). Effects of planting date on winter canola growth and yield in the southwestern U.S. American Journal of Plant Sciences 7: 201-217

Brunetti G, Senesi N \& Plaza C (2007). Effects of amendment with treated and untreated olive oil mill waste waters on soil properties, soil Humic substances and wheat yield. Journal Geoderma 138: 144-152

Chauhan A K, Singh M \& Dadhwal K S (1993). Effect of nitrogen level and row spacing on the performance of rape (Brassica napus L). Indian Journal of Agronomy 37(4): 851-853

Chris W, Anderson N \& Stewart R B (2005). Soil and foliar application of Humic acid for mustard production. Environmental Pollution pp. 254-257 
Darby H, Harwood H, Cummings E, Madden R \& Monahan S (2013). 2012 Winter canola planting date trial. University of Vermont Extension, Burlington, Vermont

Delfine S, Tognetti R, Desiderio E \& Alvino A (2005). Effect of foliar application of $\mathrm{N}$ and Humic acids on growth and yield of durum wheat. Journal of Agronomy and Sustainable Development 25: 183-191

El-Ghamry A M, Abd El-Hai K M \& Ghoneem K M (2009). Amino and Humic acids promote growth, yield and disease resistance of faba bean cultivated in clayey soil. Australian Journal of Basic and Applied Sciences 3(2): 731-739

El-Nemr M A, El-Desuki M, El-Bassiony A M \& Fawzy Z F (2012). Response of growth and yield of cucumber plants (Cucumis sativus L.) to different foliar applications of Humic acid and bio-stimulators. Australian Journal of Basic and Applied Sciences 3: 630-637

Erik B G F, Clint G S \& Lament D S (2000). Evaluation of Humic acid and other nonconventional fertilizer additiones for onion production. Malheur Experiment Station Oregon State University. Ontario, OR

Faraji A, Latifi N, Soltani A \& Rad A H S (2009). Seed yield and water use efficiency of canola (Brassica napus L.) as affected by high temperature stress and supplemental irrigation. Agricultural Water Management 96: 132-140

Farnia A (2006). Effects of drought stress on nitrogen fixation of the bacteria strains of Rhizobium japonicum and their connection with growth and yield of Soybean (Clark variety). PhD Thesis, Islamic Azad University, Science and Research Branch, Iran, pp. 150-165

Farnia A M \& Nasrollahi A (2010). Studying performance and component of chickpea cultivar affected by biological fertilizer. In: $5^{\text {th }} \mathrm{New}$ Innovations Seminar in Agriculture, Islamic Azad University of Khorasgan Branch

Farre I, Robertson M J, Walton G H \& Asseng S (2002). Simulating phenology and yield response of canola to sowing date in Western Australia. Australian Journal of Agricultural Research 53: 1155-1164

Gammelvind L H, Schjoerring J K, Mogensen V O, Jensen C R \& Bock J G H (1996). Photosynthesis in leaves and siliques of winter oilseed rape (Brassica napus L.). Plant and Soil 186: 227-236
Gan Y, Malhi S S, Brandt S A \& Mcdonald C L (2008). Assessment of seed shattering resistance and yield loss in five oilseed crops. Canadian Journal of Plant Science 88: 267-270

Hafez M M (2003). Effect of some sources of nitrogen fertilizer and concentration of Humic acid on the productivity of squash plant. Egyptian Journal of Applied Science 19(10): 293-309

Hagh-Parast M, Maleki Farahani S, Sinaki M J \& Zare G $H$ (2012). Reduction of negative effects of dry tension and stress in chickpea with the application of Humic acid and seaweed extract. Crop Under Environmental Stresses 4: 59-71

Holman J, Maxwell S, Stamm M \& Martin K (2011). Effects of planting date and tillage on winter canola. Crop Manage doi: 10.1094/CM-2011-0324 01-RS

Iqbal M, Akhtar N, Zafar S \& Ali I (2008). Genotypic responses for yield and seed oil quality of two Brassica species under semi-arid environmental conditions. South African Journal of Botany 74: 567-571

Jansinka Z, Kotecki A, Malarz W, Horodyski A, Musnicka B, Musnicki C, Jodlowski M, Budzynski W, Majkowski K, Wrobel E \& Sikora B (1989). Effect of sowing date and sowing rate on the development and seed yields of winter rape cultivars. Buile, InstitiHodowli-I, Aklimatyzacji Roslin 169: 111-119

Jenkins P D \& Leitch M H (1986). Effects of sowing date on the growth and yield of winter oil seed rape (Brassica napus L). Journal of Agricultural Science UK 107(2): 405-420

Kutcher H R, Warland J S \& Brandt S A (2010). Temperature and precipitation effects on canola yields in Saskatchewan, Canada. Agricultural and Forest Meteorology 150: 161-165

Lee C H, Shin H S \& Kang K H (2004). Chemical and spectroscopic characterization of peat moss and its different humic fractions. Journal of Korean Society of Soil \& Groundwater Environment 9: 42-51

MacCarthy P, Clapp C E, Malcom R L \& Bloom P $\mathrm{R}$ (2001). In: Humic substances in soil and crop sciences: selected readings. Soil Science Society of America, Madison, W.I, pp. 161-186

Mirzamasoumzadeh B, Salami M, Ghalichechi S \& Rouhi N (2012). A comparison study on Humic acid fertilizers effect on initial growth stages on four wheat cultivars. Annals of Biological Research 3(10): 47474750 
Rajpar I, Bhatti M B, Zia-ul-Hassan \& Shah A N (2011). Humic acid improves growth, yield and oil content of (Brassica compestris L.). Pakistan journal of Agriculture, Agricultural Engineering and Veterinary Sciences 27(2): 125-133

Rao V M, Govindasamy R \& Chanderasekaran S (2000). Effect of Humic acid on Indian mustard (Brassica juncea), var. SH-9. Current Science 6(4): 173-176

Robertson M J, Holland J F \& Bambach R (2004). Response of Canola and Indian Mustard to sowing date in the Grain Belt of North-Eastern Australia. Australian Journal of Experimental Agriculture 44: 43-52

Saadati J \& Baghi M (2014). Evaluation of the effect of various amounts of Humic acid on yield, yield components and protein of chickpea cultivars (Cicer arietinum L.). International Journal of Advanced Biological and Biomedical Research 2(7): 2306-2313

Salt D E, Prince R C, Pickering I J \& Raskin I (2001). Effect of various levels of Humic acid (HA) on the growth and yield of Indian mustard. Plant Physiology 109: 1427-1433

Salwa \& Eisa A I (2011). Effect of amendments, Humic and amino acids on increases soil fertility, yields and seeds quality of peanut and sesame on sandy soils. Research Journal of Agriculture and Biological Science 7(1): 115-125

Sani B (2014). Foliar Application of Humic acid on plant height in Canola. APCBEE Procedia 8: 82-86

Sharief A E \& Keshta M M (2000). Response of some canola Cultivars (Brassica napus L.) to different sources and levels of nitrogen fertilizers in soil affecting by salinity. Zagazig Journal of Agricultural Research 27(3): 603-616

Sparks D L (2003). Environmental Soil Chemistry, University of Delaware, pp. 82-84

Turhan H, Gül, K M, Egesel Ö C \& Kahrıman F (2011). Effect of sowing time on grain yield, oil content, and fatty acids in rapeseed (Brassica napus subsp. oleifera). Turkish Journal of Agriculture and Forestry 35: $225-234$

Ulukan H (2008). Humic acid application into field crops cultivation. Kahraman Maras Sutra Imen University, Journal of Science 11(2): 119-128

United State Department of Agriculture (USDA) (2013). Economic Research Service. http:/www.ers.usda. gov/publications/ocs-oil-crops-outlook (Access date: 04.10.2017)

Usman K, Khan S \& Ayatullah A (2016). Genotype-bysowing date interaction effects on Cotton yield and quality in irrigated condition of Dera Ismail Khan, Pakistan. Pakistan Journal of Botany 48(5): 19331944

Wang S, Wang E, Wang F \& Tang L (2012). Phenological Development and Grain Yield of Canola as Affected by sowing date and climate variation in the Yangtze River Basin of China. Crop \& Pasture Science 63: 478-488

Wang Z, Miao Y \& Li S (2015). Effect of ammonium and nitrate nitrogen fertilizers on wheat yield in relation to accumulated nitrate at different depths of soil in dry lands of China. Journal of Field Crop Research 183: 211-224 